\title{
Interpretation of enhancement factor in nonplanar field emitters
}

\author{
R. C. Smith, ${ }^{\text {a) }}$ R. D. Forrest, and J. D. Carey \\ Nanoelectronics Centre, Advanced Technology Institute, University of Surrey, Guildford GU2 7XH, United \\ Kingdom \\ W. K. Hsu \\ Department of Materials Science and Engineering, National Tsing Hua University, Hsinchu 300, Taiwan \\ S. R. P. Silva \\ Nanoelectronics Centre, Advanced Technology Institute, University of Surrey, Guildford GU2 7XH, United \\ Kingdom
}

(Received 15 September 2004; accepted 1 June 2005; published online 30 June 2005)

\begin{abstract}
A comparison of the field emission properties of exposed nanotubes lying on a tipped carbon nanorope, with the emission properties from a sharpened iron tip of similar dimensions is performed. By varying the electrode separation it is observed that the threshold field for emission for both structures decreases as the electrode separation initially increases; however, for sufficiently large electrode separations, the threshold field is observed to reach an asymptotic value. Our results show that the field enhancement factor is fundamentally associated with the electrode separation, and depending on the experimental conditions in order to obtain a true value for electric field a set of alternative definitions for enhancement factors is required. We further confirm our experimental synopsis by simulation of the local electrostatic field which gives results similar to those obtained experimentally. (C) 2005 American Institute of Physics. [DOI: 10.1063/1.1989443]
\end{abstract}

Since the first report of carbon nanotubes $(\mathrm{CNTs})^{1}$ there has been much interest in their use as electron sources in vacuum microelectronics and nanoelectronics. ${ }^{2}$ This has been brought about by considerable research in the field of carbon based cold cathodes ${ }^{3,4}$ and, in general, coupled with the ability to adjust the geometric field enhancement factor by tailoring the physical dimensions (height and radius) of the nanotubes. There are now numerous reports of electron field emission from a range of CNTs consisting of isolated single tubes, ${ }^{5}$ well ordered arrays of tubes, ${ }^{6}$ random mats, ${ }^{7}$ and, lately, from CNTs embedded in polymer composites. ${ }^{8}$ Indeed, the high aspect ratio that is found with CNTs is also found in a wide range of nanotip cold emitter materials such as Si nanowires, ${ }^{9} \mathrm{~W}$ nanowires, ${ }^{10} \mathrm{Cu}_{2} \mathrm{~S}$ nanowires, ${ }^{11}$ and nanobelts of $\mathrm{MoO}_{3}{ }^{12}$ making these nonplanar materials a creditable electron source. There have also been significant technological developments since the first report of carbon nanomaterials grown at low temperature. ${ }^{13}$ To date, both field emission from room temperature grown nanofibers ${ }^{14}$ as well as CNTs growth on plastic ${ }^{15}$ have been reported.

Despite the different material systems involved, each of these studies possess at least one fact in common. The emitter material is not a flat cathode but has features possessing high aspect ratios. Field emission measurements that are made often attempt to quantify the behavior in terms of the applied electric field and the local electric field at the nanotip, via the field enhancement factor, $\beta$. Since the local electric field required for emission will not possess a dependence on the electrode separation, $D$, one would expect that measurements of the applied threshold field would also not possess a dependence on the relative separation of the electrodes, and that the applied electric field would be given simply as $V / D$, where $V$ is the applied potential difference. In this letter we show that this is not the case for CNTs found

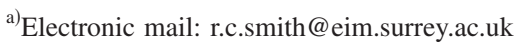

on a carbon nanorope (CNR). Furthermore, this behavior is shown not to be unique to nanotubes or nanoropes but also to applicable to all nonplaner emitters. Therefore, the implications are significant to most tip emitter structures used by scientists and technologists. Computer simulations further confirm the experimental observation. Our results show that the definition of the field enhancement factor must be modified in this situation and that this revised definition is applicable to a wide range of nonplanar cathodes.

A carbon deposit was prepared on a graphite cathode electrode in an arc discharge system in a helium atmosphere at a pressure of 500 Torr. This deposit contained multiwalled carbon nanotubes (MWCNTs) which self-assembled into bundles or ropes of micron dimensions when post-treated by grinding and subjected to ultrasonic treatment. In a Cambridge Stereoscan 250 III scanning electron microscope (SEM), the CNR has the appearance (not shown here) of high aspect ratio needles that were $1-2 \mathrm{~mm}$ in length with an end diameter of $\sim 10 \mu \mathrm{m}$. The end of the CNRs exhibited protruding MWCNTs of a few microns in length and randomly spaced, and diameters of around $100 \mathrm{~nm}$. A CNR with an end radius of $8 \mu \mathrm{m}$ was selected and adhered to a nickel tip by a conducting silver epoxy. Figure 1(a) is a SEM micrograph of the CNR with protruding CNT, and anode electrode, the inset being the anode and cathode experimental setup. An iron tip was electrolytically etched in sodium hydroxide solution to approximately the same dimensions as the CNR for comparison of field emission characteristics associated primarily with geometry.

Field emission (FE) measurements were performed in a modified Cambridge Stereoscan 250 III SEM configured with a steel probe anode with an end radius of $5 \mu \mathrm{m}$ and a FE sample stage mounted on the SEM sample stage. The FE testing system was then connected to the sample stage and anode via vacuum feedthroughs. Samples mounted on the earthed stage were tested at different sample to anode sepa- 

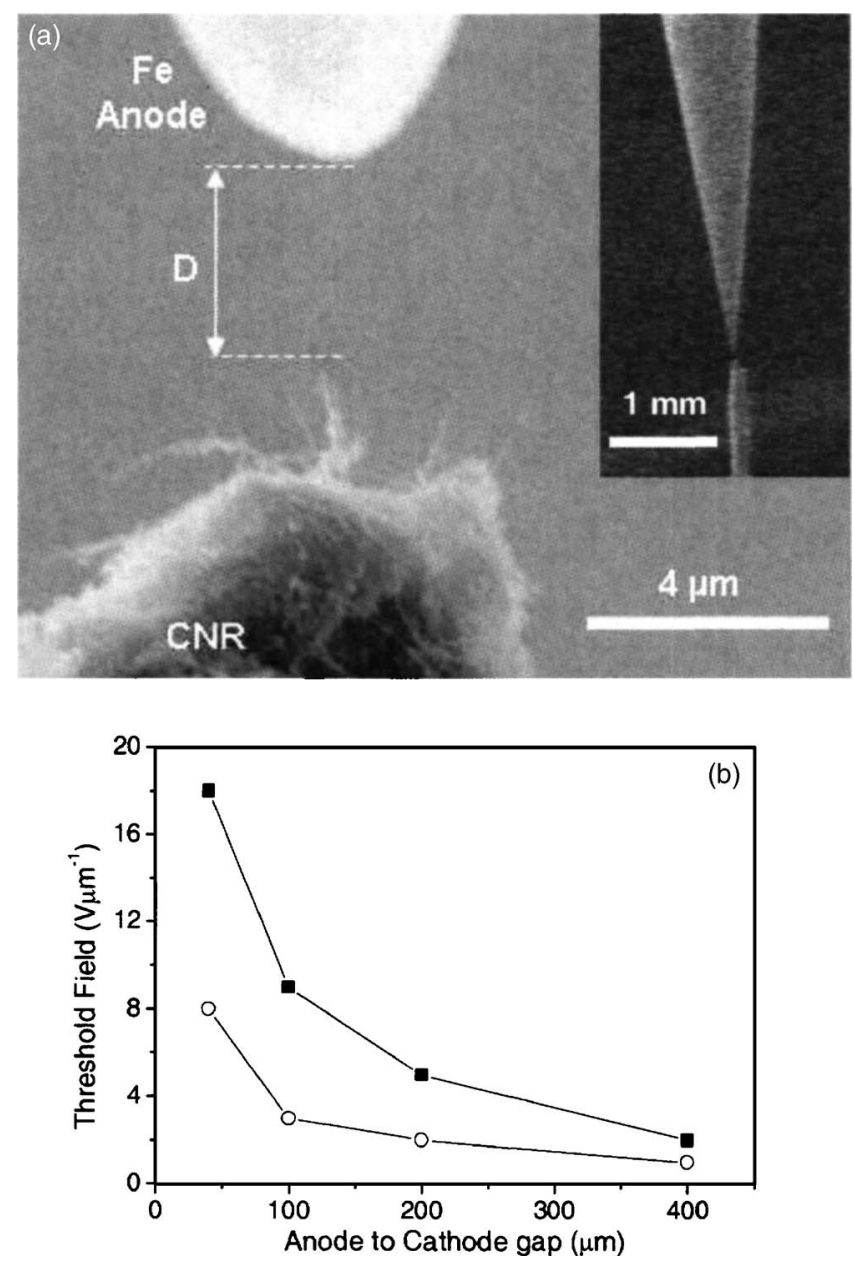

FIG. 1. (a) Scanning electron microscope image of the carbon nanorope and field emission anode. Protruding CNT are clearly visible from the CNR. The inset shows the anode and cathode experimental setup; (b) threshold field values for the carbon nanorope $(O)$ and etched iron tip $(\mathbf{\square})$ as a function of anode-cathode gap.

rations, measured accurately by the SEM, at a vacuum of better than $10^{-5}$ Torr. A positive voltage was applied to the anode and ramped from $0 \mathrm{~V}$ until an emission current of 100 $\mathrm{nA}$ was measured and then reduced to $0 \mathrm{~V}$ again. This voltage cycle was performed four times for each separate FE test and the threshold field, $\mathrm{E}_{\mathrm{T}}$, defined as the applied electric field required to extract an emission current of $1 \mathrm{nA}$ from the sample recorded. Measurements of emission from both the CNR and the etched iron tip were performed as a function of anode-sample separation. In order to remove any possible effects due to the conditioning of cathodes with time, ${ }^{16}$ the testing at different anode-sample separations was not conducted in a sequential increasing or decreasing mode; but in a random sequence. As the $I-V$ measurements were performed in a SEM, accurate measurements of the anodesample separation could be made. The SEM electron beam and collector voltage were switched off when the FE testing was performed to eliminate any influence on the measurements. While the end of the iron tip was initially jagged relative to the $\mathrm{CNR}$, the initial FE measurement resulted in it becoming smooth (on the nanoscale) and geometrically similar (on the micron scale) to the CNR. This change in morphology is attributed to ohmic heating at the tip and melting the iron due to high current densities in the sharp asperities present initially at the end of the tip. No other changes in the Downloaded 30 Mar 2009 to 131.227.178.132. Redistribution subject morphology of either sample were observed after subsequent FE testing. Figure 1(a) is a SEM image of the CNR and Fe anode. The main SEM is a higher magnification of the end of the CNR with protruding CNTs clearly visible. The anodesample separation was accurately measured by the SEM and taken as the distance between the tip of the anode and the tip of the highest protruding CNT.

Values of threshold electric field [Fig. 1(b)], were extracted from typical $I-V$ curves. In this case, the applied electric field, $\mathrm{E}_{\text {applied }}$, is defined as the applied voltage divided by the separation; $D$, between the end of the anode and cathode tips. Typical $E_{T}-D$ curves are shown in Fig. 1(b). The data in Fig. 1(b) clearly shows that the CNR has a lower value of $E_{T}$ relative to the iron tip, over the range of electrode gaps tested. Figure 1(b) also shows that, for both samples, $E_{T}$ drops with increasing electrode gap, with the rate of decrease reducing with increasing electrode gap.

Since local threshold field, $E_{T}^{\text {local }}$, can be expressed as $E_{T}^{\text {applied }} / \beta$, and the reduction of $E_{T}^{\text {applied }}$ which subsequently increases with a flattening of $\mathrm{D}$, implies that the enhancement factor must exhibit a dependence with $\mathrm{D}$. Indeed to maintain electrode geometry independence for values of $E_{T}^{\text {local }}, \beta$ must increase with $D$ before saturating. In order to test this hypothesis we have simulated; (i) a bare iron tip emitter with nonplanar anode, (ii) an iron tip emitter with nine randomly arranged CNT with a nonplanar anode and, (iii) a single CNT with a planar anode. The commercially available SILVACO ${ }^{\mathrm{TM}}{ }^{17,18}$ simulation package was employed to simulate the emission from the experimental setup. The FE properties of a metallic cathode with a semicircular tip of radius of $4 \mu \mathrm{m}$ (mimicking the etched iron tip), and an identical cathode but with seven metallic randomly positioned spikes with approximate lengths and widths of $2 \mu \mathrm{m}$ and $100 \mathrm{~nm}$, respectively (mimicking the CNR). Simulated FE measurements were made between the cathodes and an iron anode with a tip radius of $5 \mu \mathrm{m}$ using anode tip to CNT/iron probe tip gaps of $10,20,40,100,200$, and $400 \mu \mathrm{m}$. The variation of $\mathrm{E}_{\mathrm{T}}$ vs separation for an undecorated tip and a tip decorated with seven nanotubes is shown in Fig. 2(a). The decreasing trend of threshold field is the same as that of the experimental work.

We further proceeded to simulate an isolated nanotube and vary anode to CNT separation, recording the threshold field to see if the effects experienced on isolated tubes are similar to randomly arranged ones. Metallic nanotubes, which allow us to ignore the effects of field penetration, of heights 2,4 , and $6 \mu \mathrm{m}$ with constant $200 \mathrm{~nm}$ radius were simulated with anode to cathode separation increased until an emission current was no longer observed from the single nanotube. The value of $\mathrm{E}_{\mathrm{T}}$ was seen to decrease as the anode electrode was moved away from the tip of the tube, decreasing to a saturated value, as shown in Fig. 2(b), which is similar to the trend seen experimentally for the protruding nanotubes of the CNR and etched iron probe, and for simulated CNR and iron probe. Our results clearly point to a need for an understanding of the variation of $\beta$ with $D$. Figure 3 shows the variation of the enhancement factor $\beta_{1}$, (closed shapes) for an applied field of $V / D$. It is clear that $\beta_{1}$ decreases with $D$ before flattening out. This behavior is clearly at odds with the required behavior discussed in the literature of a constant threshold field. As an alternative definition we define $\beta_{2}$ (open shapes), as the enhancement factor when the applied field is taken as $\mathrm{V} /(D-h)$. In this way we are conto AIP license or copyright; see http://apl.aip.org/apl/copyright.jsp 

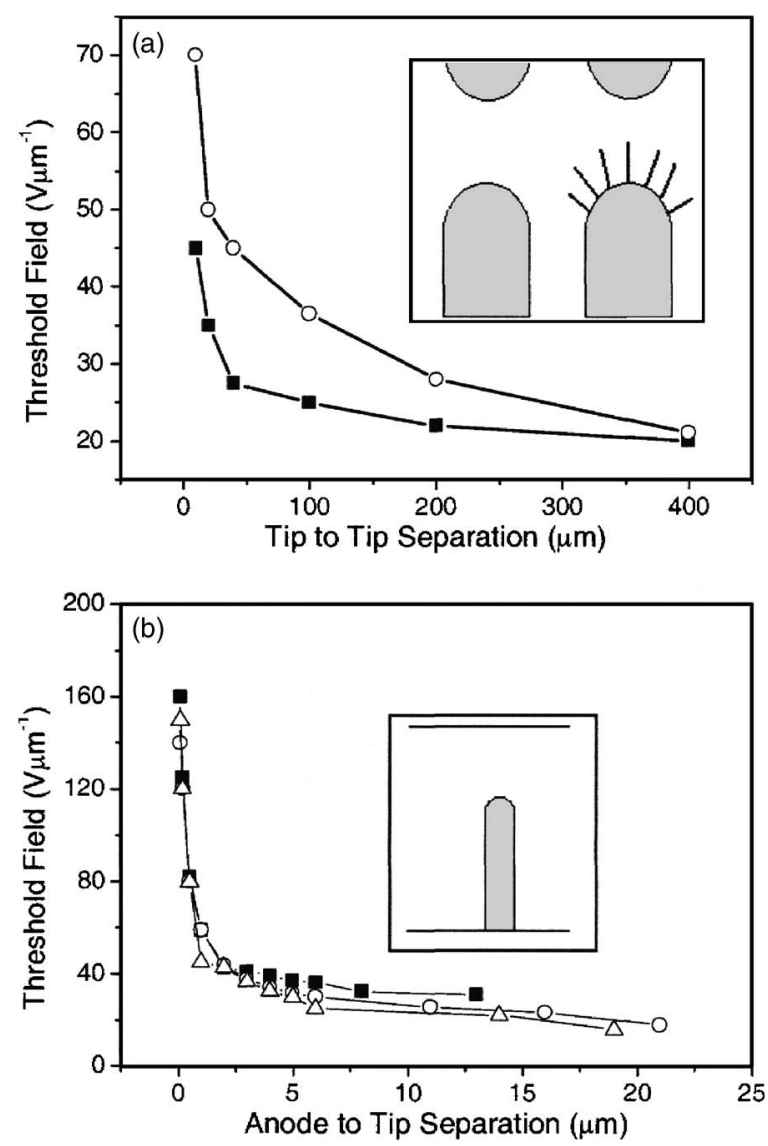

FIG. 2. (a) Threshold field plotted as a function of anode-cathode gap from the simulation data for both iron tip and seven randomly placed nanotubes. Inset is simulated iron tip and carbon nanorope with anode configuration; (b) threshold field plotted against anode to cathode separation for simulated nanotubes of height $2(\mathbb{\square}), 4(\bigcirc)$, and $6 \mu \mathrm{m}(\triangle)$ with radius $0.2 \mu \mathrm{m}$. Inset is simulated nanotube with anode configuration.

sidering the separation to be between tip of the highest protruding CNT, and anode. This definition is acceptable as it mimics the case of the local field distribution between a ball anode and planer cathode. For large values of $\mathrm{D}$, the curves converge in the limit of $D \gg h$ to a value depicted by the geometric properties of the emitter alone and not the location of the anode. Three sets of data are presented for nanotubes of height 2, 4, and $6 \mu \mathrm{m}$ with a radius of $200 \mathrm{~nm}$. The values

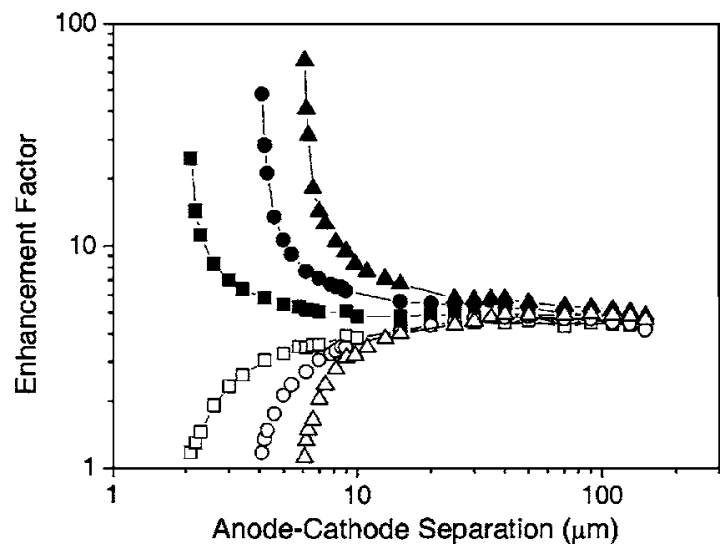

FIG. 3. Variation of enhancement factor for a nanotube of $\mathrm{h}=2(\boldsymbol{\square})$, ( $\square$ ), $\mathrm{h}=4(\bullet),(\bigcirc)$, and $\mathrm{h}=6 \mu \mathrm{m}(\boldsymbol{\Delta}),(\triangle)$ each with radius $=200 \mathrm{~nm}$. Closed symbols are associated with $\beta_{1}$ and open symbols with $\beta_{2}$. of $\beta_{1}$ and $\beta_{2}$ can be seen to converge to similar values for a given nanotube. This highlights that at sufficient separation (when $D \gg h$ ) the enhancement factor is dependent on the geometrical properties of the nanotubes alone and not $D$.

The results of the experiments, supported by these simulations demand that for a nonplanar emitter and alternative definition of field enhancement factor must be considered. Both expressions for $\beta_{1}$ and $\beta_{2}$ can be expressed as

$$
\beta=\frac{E_{\text {local }}}{V /(D-\alpha h)},
$$

where $0<\alpha<1$ encapsulates all possible configurations. The simulation results tie in with the increased threshold field seen in both experimental and simulated results. The increase in threshold field and decrease in $\beta$ has also been shown experimentally elsewhere. ${ }^{19}$ Note that for planar cathodes and planar anodes we recover the usual definition of $\beta$.

In conclusion, we have shown that for tip based field emitters the location of the anode plane significantly affects the field emission capabilities of the emitter, with an increase in threshold field as the anode electrode approaches the tip of the emitter. Furthermore, the geometric field enhancement factor for the emitter is greatly reduced when the anode to tip separation is decreased.

The authors would like to thank the EPSRC Portfolio Partnership and Carbon Based Electronics Programs for funding this research.

${ }^{1}$ S. Iijima, Nature (London) 56, 354 (1991).

${ }^{2}$ A. G. Rinzler, J. H. Hafner, P. Nikolaev, L. Lou, S. G. Kim, D. Tománek, P. Nordlander, D. T. Colbert, and R. E. Smalley, Science 269, 1550 (1995).

${ }^{3}$ J. E. Jaskie, MRS Bull. 21, 59 (1996).

${ }^{4}$ W. B. Choi, N. S. Lee, W. K. Yi, Y. W. Jin, Y. S. Choi, I. T. Han, H. Y. Jang, H. Y. Kim, J. H. Kang, M. J. Yun, S. H. Park, S. G. Yu, J. E. Jang, J. H. You, and J. M. Kim, J. Soc. Inf. Disp. 1, 59 (2000).

${ }^{5}$ G. Pirio, P. Legagneux, D. Pribat, K. B. K. Teo, M. Chhowalla, G. A. J. Amaratunga, and W. I. Milne, Nanotechnology 13, 1 (2002).

${ }^{6}$ J. M. Bonard, H. Kind, T. Stockli, and L. A. Nilsson, Solid-State Electron. 45, 893 (2001).

${ }^{7}$ I. Alexandrou, E. Kymakis, and G. A. J. Amaratunga, Appl. Phys. Lett. 80, 1435 (2002).

${ }^{8}$ C. H. Poa, S. R. P. Silva, P. C. P. Watts, W. K. Hsu, H. W. Kroto, and D. R. M. Walton. Appl. Phys. Lett. 80, 17 (2002).

${ }^{9}$ K. W. Wong, X. T. Zhou, F. C. K. Au, K.-L. Lai, C. S. Lee, and S. T. Lee, Appl. Phys. Lett. 75, 2918 (1999).

${ }^{10}$ Y.-H. Lee, C.-H. Choi, Y.-T. Jang, E.-K. Kim, and B.-K. Ju, Appl. Phys. Lett. 81, 745 (2002).

${ }^{11}$ J. Chen, S. Z. Deng, and N. S. Xu, Appl. Phys. Lett. 80, 3620 (2002).

${ }^{12}$ B. Li, Y. Bando, D. Golberg, and K. Kurashima, Appl. Phys. Lett. 81, 5048 (2002).

${ }^{13}$ B. O. Boskovic, V. Stolojan, R. U. A. Khan, S. Haq, and S. R. P. Silva, Nat. Mater. 1, 165 (2002).

${ }^{14}$ R. C. Smith, J. D. Carey, C. H. P. Poa, D. C. Cox, and S. R. P. Silva, J. Appl. Phys. 95, (2004).

${ }^{15}$ S. Hofmann, C. Ducati, B. Kleinsorge, and J. Robertson, Appl. Phys. Lett. 83, 4661 (2003).

${ }^{16}$ J. D. Carey and S. R. P. Silva, Appl. Phys. Lett. 78, 347 (2001).

${ }^{17}$ Silvaco International.

${ }^{18}$ Modifications to the Fowler-Nordheim model had to be performed as this model was originally designed for use in MOSFET devices calculating emission current between silicon and silicon oxide layers. Therefore, the emission barrier is defaulted to $2 \mathrm{eV}$. For emission from CNTs into a vacuum an emission barrier of approximately $4.5 \mathrm{eV}$ is used. This is achieved by modifying the two variables, f.ae and f.be which control the Fowler Nordheim model within the Silvaco package.

${ }^{19}$ J. M. Bonard, Diamond Relat. Mater. 11, 763 (2002). 